\title{
Cognitive Science: Bridging the Gap between Machine and Human Intelligence
}

\author{
Komal \\ Assistant Professor \\ Amity University Haryana
}

\begin{abstract}
Cognitive Science is the interdisciplinary study of concepts and theories related to the fields of psychology, neuroscience, philosophy, computer science, artificial intelligence, humanities and linguistics. A lot of research has been done in the area of machine learning and artificial intelligence which aims to inculcate human thinking, sensing and decisive capabilities into machines. However, such systems lack in various aspects of human behavior and intelligence such as emotions, intuitions, understanding of linguistics and learning acquired from different happenings and incidents occurred in the past. Cognitive science is an emerging field which intends to fill the existing gap in this area to build smarter systems. This paper discusses the advancements in the field of cognitive science, various models and their applicability to different problem domains. The paper also suggests framework of HAICS, a computer system having human alike intelligence.
\end{abstract}

\section{Keywords}

Cognitive Science, Intelligent Systems, Artificial Intelligence, Knowledge Representation, Neuroscience.

\section{INTRODUCTION}

Researchers over a decade have been trying really hard to understand the complex mechanisms and representations of human mind and why not human beings are considered the most intelligent of all living beings. All inventions of today's world have been produced by a number of intelligent human brains. One of significant invention is the field of robotics where machines are devised to imitate human behavior and perform tasks as good as a human being. Artificial Intelligence, neural networks, knowledge representation and experts systems are the subject areas related to computer science that try to simulate one or the other behavior and representation as exhibited by human brain.

Today, cognitive science has emerged as a superset of artificial intelligence which has adopted concepts, theories and methods of multiple disciplines other than those of computer science. It includes psychology, linguistics, neuroscience, philosophy, sociology in addition to artificial intelligence, neural networks and expert systems. Psychologists advocate that mental state of a person can be understood by his behavioral characteristics. Linguists deals with language processing (i.e. to learn and understand the context of language) in order to obtain useful information. Neuroscience is a field that studies the biological aspects of human brain. Philosophy[1] contributes to cognitive science by answering generic queries in scientific investigations and by suggesting how brain needs to work. Sociologists believe that the cause of a particular is mostly the outside world. It means outside social factors are responsible and they affect the decision-making process.

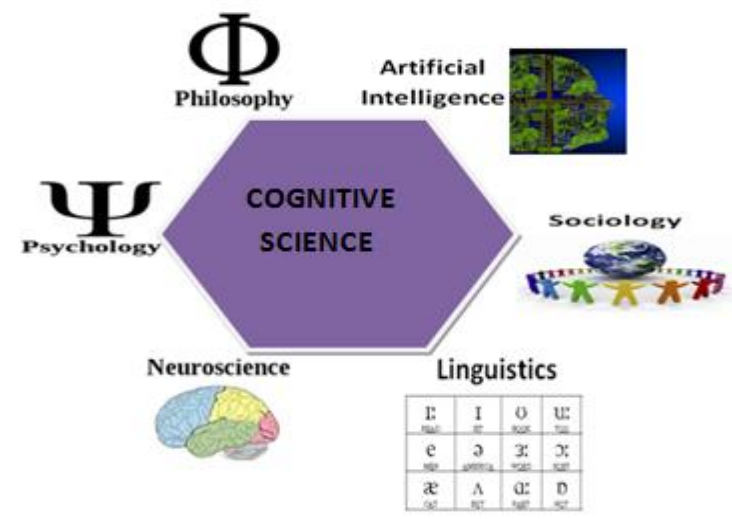

Fig 1: Multidisciplinary approach of cognitive science

Cognitive science approach to solve a problem include following steps- First and foremost thing is to ensure an appropriate representation of problem including the critical features and avoiding irrelevant ones [2]. Second step is to discover the goal state of the problem that. Third step applies different approaches and methods, somewhat like hit and trial, to discover alternative ways to solve the problem. Fourth step is to find the feasibilities of identified mechanisms with respect to the constraints. Last step is to apply the feasible methodologies which facilitate the process of decision making and try to find an optimal solution.

Though the processes of cognitive science make use of preestablished theories, past experiences, various logics and structures for problem representation and its solution; there are some indescribable phenomena which may be used at different stages of problem-solving such as intuition (an instant solution to a problem without knowing and explaining the steps applied), insight (deeply analyzing the problem state after continuous failures) and inspiration (an inward push or trust that solution will be found even after failures) [3].

The paper is structured as follows: Section 2 discusses various models used for information representation, evaluation of various problems and finding their solutions. Every model works on certain theories, which have been discussed in the paper. Section 3 presents a framework and characteristics of HAICS (Human Alike Intelligent Computer System).Section 4 concludes the paper with scope of future developments.

\section{MODELS OF COGNITIVE SCIENCE}

Cognitive science works broadly on three modelsComputational model, Mathematical model and VerbalConceptual model as shown in figure below. 


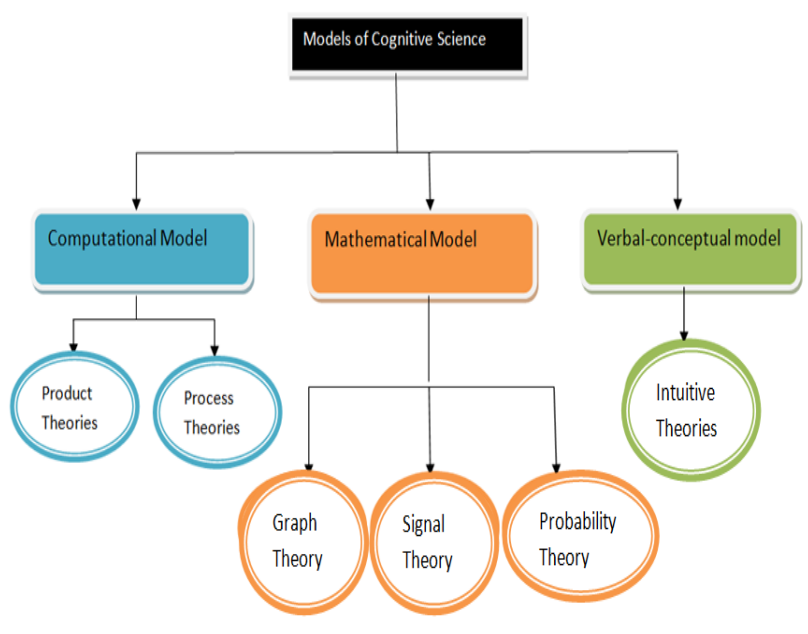

Fig 2: Models \& Theories of Cognitive Science

\subsection{Computational Model}

Computational model provides specification of various operations and processes in detail using algorithms (which includes precise and step-wise sequence of instructions). Since computational models study intrinsic and finer details of brain functions, they provide precision as well as clarity. Computational model [4] can be further classified asQuantitative (working with numerical and precise data), Qualitative (working with non-numerical and relative aspects of information). Different theories are related to computational model (Sun 2005) which are discussed as follows.

\subsubsection{Product Theory-}

Product theories are also known as "black box theories" [4] and they check the accountability of processes based on the output achieved in respect with the input requirements as suggested earlier. Product theory doesn't go into the intrinsic details of processes and mechanisms; instead it is concerned with what input is to be provided to a process and what output (product) is achieved.

\subsubsection{Process Theory}

Process theory [4] tries to relate and understand the complexity and diversity of processes. It is also known as "white box theory". It inspects the inner logic and detail of components of the processes. Each process makes a discrete and random selection of parameters from the available options, depending upon a scenario and performs various computations to achieve a desired result.

\subsection{Mathematical Model}

Mathematical model showcases changing aspects and dynamic state of the system. It represents relationship between entities in the problem domain using mathematical equations. Mathematical model comprises of various theories and components [5] such as algebra, probability, signal \& control theory, gaming, graphs etc.

\subsubsection{Graph Theory}

Graph theory, rooted deep into mathematics, is an approach to model relationships between various processes, objects and information blocks in psychological, sociological, biological and informational systems. Graphs are a powerful way to relate complex structures and pieces of information in a pictorial way. Edges of graph show associations and possible paths between entities.

\subsubsection{Probability Theory}

Probability theory makes an important base of all decision making processes. Human brain also makes use of probability to evaluate random and non-deterministic events. Bayes' theorem of probability theory is a significant approach for drawing conclusions using current as well as former interpretations.

\subsubsection{Signal Theory}

Signal theory is applied heavily to biological processes including signals as generated by brain. Signal theory illustrates how two processes exchange their genuine information. According to signal theory, information is coded into signals and sending process decides on which information to send and how. Whereas, receiving process finds a way to interpret the received signal.

\subsection{Verbal-Conceptual Model}

Verbal-Conceptual model provides description of entities, relationship between them and functions operating on their data in natural languages. Verbal-Conceptual model presents abstraction of information. It differentiates the contexts of information from prescribed information and removes any type of biasness (if present) for drawing conclusions. VerbalConceptual model requires knowledge of grammar, syntax of language, logical reasoning and inferential processes. VerbalConceptual models are generally governed by intuitive theory.

\subsubsection{Intuitive Theory}

Intuitive theory [6] aims to reason about mental state (which includes perceptions, beliefs, desires, insecurities, emotions etc.) of different people and draw significant inferences. Intuitive theory makes use of various skills like elementary prediction, justification and improvisation based on new facts and evidences. According to intuitive theory, any belief can be proved false by a person by providing a valid proof. Human brain uses true beliefs to fulfill the goals and also to overcome risks and insecurities.

Another important role of intuitive theories is to elucidate inferences drawn by assuming \& suggesting states of unobserved variables, from which inferences follow. It means consistency between prediction and depiction.

\section{HUMAN ALIKE INTELLIGENT COMPUTER SYSTEM (HAICS)}

The objective of all researches done so far in the field of cognitive science is to empower computer systems with human intelligence capabilities which will support and supplement human brain requirement. Such systems can be deployed at places where human existence is not feasible like nuclear reactors, adverse atmospheric conditions, combat situation etc. This paper also endeavors to sketch a framework for designing computer systems with brains.

In order to provide learning and thinking abilities to a computer same as that of human brain, various stages of human brain development must be understood. The different stages of brain development are as depicted in figure 3. Basically, human brain also takes a significant time (2-7 years) to upgrade its information base, create knowledge representations, understand language syntax, establish beliefs, construct aspirations, relate happenings, analyze problems, imbibe creativity and develop independent, rational decision making capability. 


\begin{tabular}{|c|c|c|}
\hline Stage & Age & Description \\
\hline Sensorimotor & $0-2$ & Senses active \\
\hline Preoperational & $2-6$ & $\begin{array}{c}\text { Language processing } \\
\text { Self-centered approach }\end{array}$ \\
\hline $\begin{array}{c}\text { Concrete } \\
\text { Operational }\end{array}$ & $6-12$ & $\begin{array}{c}\text { Many viewpoints } \\
\text { Logical thinking }\end{array}$ \\
\hline $\begin{array}{c}\text { Formal } \\
\text { Operational }\end{array}$ & $12-17$ & $\begin{array}{c}\text { Think conceptually } \\
\text { Hypothesis \& Reasoning }\end{array}$ \\
\hline Dialectic & $17-21$ & $\begin{array}{c}\text { Inferred correlation } \\
\text { Problem solving } \\
\text { Decision making }\end{array}$ \\
\hline
\end{tabular}

Fig 3: Development stages of human brain [7]

By studying the development phases of human brain, one can clearly identify that intelligence is not an impetuous process and can be acquired by improvising and substituting prior knowledge and experiences. The proposed framework of HAICS (Human Alike Intelligent Computer System) requires various components (in addition to machine computational capabilities) which are yet to be implemented with computer systems and are explained further.

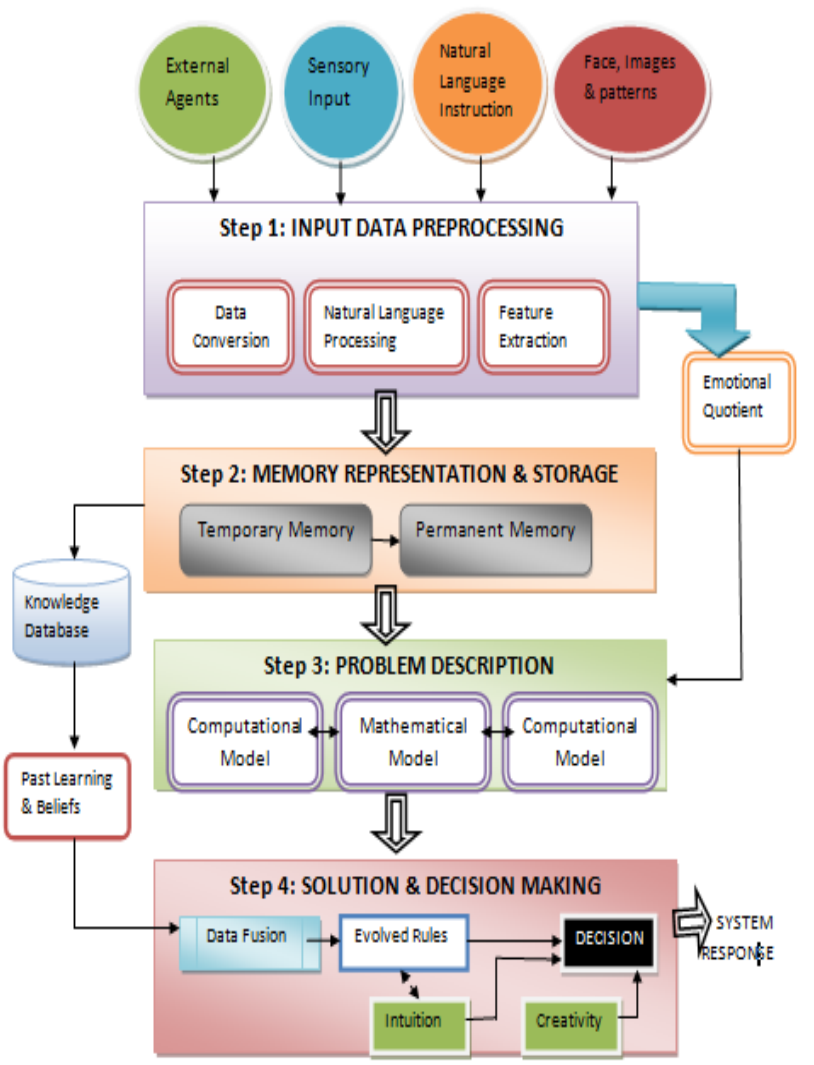

\section{Fig 3: Framework of Human Alike Intelligent Computer System}

a) Sovereign thinking- It is advocated by many sociologists that human thinking is greatly affected by outside environment conditions. Psychologists have given proof through experiments that human brain is an autonomous[8] entity which does take input from external factors but the representation, computation and analysis of the stored information and acquired input is completely independent and diverse. HAICS should be self-sufficient to solve any type of problem.

b) Rational decision- The ability to take a decision which is derived from logics and beliefs of self and is not influenced by personal interest or any biasing is assumed to be "rational". Optimism is also achieved by a rational thinking process[8]. Though decisions taken by human beings are widely affected by their emotions and thus deemed as "irrational". Irrational decisions may lead to serious consequences. Hence HAICS are expected to exhibit rational decision capabilities.

c) Robust- No matter how inconsistent and uncertain information is available for solving a problem, there is always a feasible solution provided and it doesn't bother other components and solutions, thus achieving a stable state.

d) Creativity- One of the most wonderful ability of human mind is creativity [9] i.e. finding completely innovative solution which is not influenced by a priori knowledge and solution base for similar problems. Computer systems designed so far are missing the creativity component as they use pre-programmed logics and pre-established notions for finding a solution and doesn't jump to unexplored assumptions and logics on its own. Creativity is not just limited to computational and problem finding arena, it has a wider scope. Harold Cohen's[10] designed computer program, AARON, to create ART is a significant contribution.

e) Exception handling ability- Sometimes we face completely unforeseen problems having no base. Intelligent systems are programmed to deal with all such problems in a specified way (i.e. preventing system crash or failure) to continue working normally in cases otherwise. However, human intelligence finds a unique way of handling each exception (which is again contributed to creativity). It is focused to find a solution to the issue and doesn't skip that issue, as done by computer systems.

f) Automated reasoning- There has always been a debate whether the computer reasoning and decision making process should be controlled or automated [11].It is suggested that HAICS must exhibit automated representation of a problem by linking its inputs to pre-stored information blocks just like a human mind. Once the network representation is ready, further processing to find optimal paths to a decision goal must be automatically triggered.

g) Natural language processing- Mostly computer programs are designed in high-level programming languages. Computer systems understand and interpret only the commands give in that format and do not understand human languages and hence can't work for queries put up in natural language by any person. HAICS must have complete understanding of natural language so that it can derive relevant input from the text provided by user and performs required task.

h) Emotional Quotient- Since human is emotion-driven being. HAICS must be able to understand and relate to the emotional state of human mind and coordinate with it to avoid irrational and inclined decisions. Hence, computer systems having a balanced emotional quotient will be able to cheer up people in depressing states.

i) Pattern recognition and retrieval-The other gap between computer system memory and human memory is identification, storage, association and retrieval of patterns, images and pictorial representations. Computer systems store 
information in registers, accumulators and keyword search is used to find associated information in the memory[12]. HAICS is expected to perform pattern recognition and retrieval as effortlessly and efficiently as human brain does.

j) Intuitive behavior for indecisive cases- Intuition is another indescribable aspect of human intelligence[13]. There are certain situations where computations and logics fail. Intuition enables a decision to be taken in the confusing scenarios without even telling why and how that decision was attained. However, it is often argued that human intuitions may cause wrong decisions as they are based on certain pre-assumed conditions where machine intelligence with calculative process is more beneficial, for example, in syndrome diagnosis [14].

\section{CONCLUSION AND FUTURE SCOPE}

Cognitive science is a paradigm shift for a decade of research focused on the study of human brain using the conceptual knowledge of a particular discipline. Cognitive science has emerged as a field of combinatorial study of diverse discipline ideas making the study of mind complex but more realistic. The suggested HAICS framework put forward the dimensions of human intelligence which are yet to be applied to machine intelligence. With further advancements in the field of cognitive science, we will be able to achieve synchronization between machine and human intelligence. There is no denying the fact that computers can become closest friends for humans in the near future.

The proposed framework can be considered a base for the enhancing the capability of intelligent systems and it also suggests a number of areas where lot of research needs to be done. The future scope of this proposed framework would be finding ways to implement the suggested capabilities and also testing the efficiency for different problem scenarios in comparison to human brain outputs.

\section{REFERENCES}

[1] Paul Thagard, "Why cognitive science needs philosophy and vice-versa", Topics in Cognitive Science 1 (2009), Cognitive Science Society, 2009, pp 237-254

[2] Antonio Hernando, Luis de Ledesma, Luis M. Lalita, "An approach to representation changes while executing problem solver intelligent systems", Proc. of $6^{\text {th }}$ IEEE International Conference on Cognitive Informatics (ICCI’07), 2007, pp 35-42.

[3] H.A. Simon, "Explaining the Ineffable: AI on the topics of Intuition, Insight and Inspiration", Proc. Of $14^{\text {th }}$ International Joint Conference on Artificial Intelligence, 1995, pp 939-948.

[4] Ron Sun, "Intoduction to Computational Cognitive Modeling", The Cambridge Handbook of Computational Psychology, Cambridge University Press, 2012, pp 3-20.

[5] Daniel Andler, "Mathematics in Cognitive Science", Probabilities, Laws and Structures, Springer, Dorrecht, 2012, pp 363-377.

[6] Noah D. Goodman, Chris L. Baker, Elizabeth Baraff Bonawitz, Vikash K. Mansinghka, Alison Gopnik, Henry Wellman , Laura Schulz , Joshua B. Tenenbaum, "Intuitive theories of mind: A Rational Approach to False Belief", Proc. of $28^{\text {th }}$ Annual Conference of Cognitive Science Society, pp 13821387.

[7] William J. Hudspeth, Karl H. Pribram, "Stages of Brain and Cognitive Maturation", Joumal or Educational Psychology, 1990, Vol 82. No.4, pp 881884.

[8] Pat Langley, John E. Laird, Seth Rogers, "Cognitive Architectures: Research Issues and Challenges", Cognitive Systems Research, Vol. 10, Issue 2, June 2009, pp 141-160.

[9] Samantha Luber, "Cognitive Science Artificial Intelligence: Simulating the human mind to achieve goals", IEEE Explore, 2011, pp 207-210.

[10] Louise Sundararajan, "Mind, Machine, and Creativity: An Artist's Perspective", The Journal of Creative Behavior, Vol. 0, Issue 0, 2013, pp 1-16.

[11] Ben R. Newell, "Cognitive processes, models and metaphors in decision research", Judgement and Decision Making, Vol. 3, No. 3,March 2008, pp 195204

[12] Bernard Widrow, "Cognitive Memory and its Applications", Proc. $9^{\text {th }}$ Internaional Conference on Cognitive Informatics (ICCI'10), IEEE Explore, 2010.

[13] Jitesh Dundas, David Chik, "Implementing Humanlike Intuition Mechanism in Artificial Intelligence", http://arxiv.org/ftp/arxiv/papers/1106/1106.5917.pdf.

[14] Oivind Braaten, Johannes Friestad, "Syndrome Diagnosis: Human Intuition or Machine Intelligence?", The Open Medical Informatics Journal, 2008, Vol. 2,pp 149-159. 